10-1-1985

\title{
The Temporary Capture of Light by a Dielectric Film
}

James A. Lock

Cleveland State University, j.lock@csuohio.edu

Follow this and additional works at: https://engagedscholarship.csuohio.edu/sciphysics_facpub

Part of the Physics Commons

How does access to this work benefit you? Let us know!

\section{Publisher's Statement}

Copyright 1985 American Association of Physics Teachers. The article appeared in American Journal of Physics 53 (1985): 968-971 and may be found at http://aapt.scitation.org/doi/abs/ $10.1119 / 1.14013$

\section{Original Citation}

Lock, James A. "The Temporary Capture of Light by a Dielectric Film." American Journal of Physics 53 (1985): 968-971.

\section{Repository Citation}

Lock, James A., "The Temporary Capture of Light by a Dielectric Film" (1985). Physics Faculty Publications. 99.

https://engagedscholarship.csuohio.edu/sciphysics_facpub/99

This Article is brought to you for free and open access by the Physics Department at EngagedScholarship@CSU. It has been accepted for inclusion in Physics Faculty Publications by an authorized administrator of

EngagedScholarship@CSU. For more information, please contact library.es@csuohio.edu. 
James A. Lock

When a wave packet of light passes through a dielectric film it is found that at a transmission maximum, the group velocity of the light wave packet within the film attains its minimum value Similarly, at a transmission minimum, it is found that the group velocity of the light wave packet within the film attains its maximum value. The measurability of this effect is also discussed.

\section{INTRODUCTION}

It is well known that the speed of a monochromatic light ray propagating freely through a medium whose index of refraction is $n$ is $c / n$. However, the idealization of having truly freely propagating monochromatic light is never realized in practice. The source which produces the light must at some time be turned on and off. As a result, the source generates a wave packet of light with its corresponding spectrum of frequencies. Further, sooner or later the light encounters boundaries. The boundary conditions which these impose upon the wave equation cause the propagation not to be totally free. The modifications on the wave propagation introduced by either or both of these limitations to the usual idealization may be substantial. Such is the case for the propagation of electromagnetic radiation within a wave guide, where the boundary conditions for the air-metal interfaces give rise to the frequency dependence of the speed of light within the wave guide and give rise to the existence of a cutoff frequency. A more subtle example is provided by having a wave packet of light, initially in a region whose index of refraction is $n_{1}$, incident upon a film of thickness $d$ whose index is $n_{2}$. In this situation it is the combination of the boundary conditions and the nonmonochromatic frequency spectrum which cause modifications in the effective speed of light within the film from the ideal value of $c / n$.

The usual interpretation of transmission through a thin film is that the incident light suffers an infinite series of partial transmissions and reflections from the near and far boundaries of the film. ${ }^{1.2}$ The total transmitted amplitude is the sum over the partial transmissions, the jth of which occurs after $2 j-2$ internal reflections. In particular, for a normally incident plane wave whose initial amplitude is $\boldsymbol{A}_{i}\left(k_{1}\right)$, the amplitude of the $j$ th partial transmission is

$$
\begin{aligned}
A_{!}^{(j)}\left(k_{1}\right)= & A_{i}\left(k_{1}\right)\left[4 n_{1} n_{2} /\left(n_{1}+n_{2}\right)^{2}\right] \\
& \times\left[\left(n_{2}-n_{1}\right) /\left(n_{2}+n_{1}\right)\right]^{2 j-2} \\
& \times \exp \left[i k_{2} d(2 j-1)\right] \exp \left(-i k_{1} d\right)
\end{aligned}
$$

and the total transmitted fraction of the initial wave amplitude is ${ }^{3}$

$$
\begin{aligned}
t\left(k_{1}\right) & =\sum_{j=1}^{\infty} \frac{A_{1}^{(j)}\left(k_{1}\right)}{A_{i}\left(k_{1}\right)} \\
& =\left[1+\left(n_{1}^{2}-n_{2}^{2}\right)^{2} \sin ^{2}\left(n_{2} k_{1} d / n_{1}\right) / 4 n_{1}^{2} n_{2}^{2}\right]^{-1 / 2} e^{i \delta_{i}\left(k_{1}\right)}
\end{aligned}
$$

where the phase shift $\delta\left(k_{1}\right)$ is

$$
\delta\left(k_{1}\right)=\arctan \left(\frac{n_{1}^{2}+n_{2}^{2}}{2 n_{1} n_{2}} \tan \frac{n_{2} k_{1} d}{n_{1}}\right)-k_{1} d .
$$

However, the wave equation for electromagnetic waves normally incident upon a thin film with the associated boundary conditions for the electric and magnetic fields is formally identical to the Schrödinger equation for a particle incident upon a potential barrier or well, with the associated boundary conditions on the wavefunction and its first spatial derivative. For this second situation, at a scattering resonance, the wavefunction is interpreted as being captured temporarily by the potential and then later released. ${ }^{4}$ The particle emerges from the potential region substantially later than the classical transit time through it and thus its effective velocity through the potential region is slower than what would have been expected on classical grounds. Since the differential equations and boundary conditions for the electromagnetic and quantum mechanical situations are identical formally, the same delay phenomenon should also occur for light.

\section{THE TRANSMITTED AND REFLECTED WAVE PACKETS}

We consider an incident wave packet of light in the exterior region whose amplitude spectrum $A_{i}\left(k_{1}\right)$ is peaked about some dominant wave vector $\bar{k}_{1}$. The electric field incident upon the thin film is then

$$
\begin{aligned}
E_{1}\left(x-\frac{c}{n_{1}} t\right) & =\int_{0}^{x} d k_{1} A_{i}\left(k_{1}\right) \exp \left[i k_{1}\left(x-\frac{c}{n_{1}} t\right)\right] \\
& =\exp \left\{i \bar{k}_{1}\left[x-\left(c / n_{1}\right) t\right]\right\} F_{A}\left[x-\left(c / n_{1}\right) t\right] .
\end{aligned}
$$

where the complex exponential represents the phase variation of the dominant wave vector in the wave packet and where $F_{A}$ is the Fourier transform of the frequency spectrum and represents the amplitude modulation of the dominant wave vector. The amplitude of the $j$ th partial transmission is

$$
\begin{aligned}
E_{t}^{(j)}(x, t)= & \int_{0}^{\infty} d k_{1} A_{1}^{\prime j}\left(k_{1}\right) \exp \left[i k_{1}\left(x-\frac{c}{n_{1}} t\right)\right] \\
= & {\left[\frac{4 n_{1} n_{2}}{\left(n_{1}+n_{2}\right)^{2}}\left(\frac{n_{2}-n_{1}}{n_{2}+n_{1}}\right)^{2 j-2}\right] } \\
& \times \exp \left\{i \overline { k } _ { 1 } \left[x-\left(c / n_{1}\right) t\right.\right. \\
& \left.\left.+(2 j-1)\left(n_{2} d / n_{1}\right)-d\right]\right\} \\
& \times \mathscr{F}_{A_{i}}\left[x-\left(c / n_{1}\right) t+(2 j-1)\left(n_{2} d / n_{1}\right)-d\right] .
\end{aligned}
$$

This result behaves in a totally expected way. The initial pulse shape $\mathscr{F}_{A_{1}}$ is undistorted in the $j$ th partial transmission but its strength is attenuated by the factor in the large 
square brackets. If the initial pulse shape $F_{A}$ is peaked about the value of zero for its argument, we then have

$$
x=\left(c / n_{1}\right) t
$$

for the average position of the incoming wave, and the $j$ th outgoing wave is peaked about the position

$$
x=\left(c / n_{1}\right) t-(2 j-1)\left(n_{2} d / n_{1}\right)+d .
$$

This makes intuitive sense since the $j$ th transmission has made $2 j-1$ passes through the film traveling a distance of $(2 j-1) d$. This takes the time $(2 j-1) n_{2} d / c$. At this time, an undelayed wave traveling with the speed $c / n_{1}$ has traveled the distance $(2 j-1) n_{2} d / n_{1}$, but the $j$ th reflected wave is at the position $x=d$. It trails the undelayed wave by the distance $(2 j-1) n_{2} d / n_{1}-d$.

When the partially transmitted waves are superposed to give the total transmitted wave packet

$$
\begin{aligned}
E_{t}(x, t)= & \sum_{j=1}^{\infty} E_{t}^{(\lambda)}(x, t)=\int_{0}^{\infty} d k_{1} A_{i}\left(k_{1}\right) t\left(k_{1}\right) \\
& \times \exp \left\{i k_{1}\left[x-\left(c / n_{1}\right) t\right]\right\},
\end{aligned}
$$

two interference effects occur. The first interference effect (which is the usual one considered) is between the phases of the dominant wave vector in consecutive partial transmissions. For constructive interference of the dominant wave vector and a transmission maximum, this phase difference $2 n_{2} \bar{k}_{1} d / n_{1}$ must be equal to an integer number of cycles,

$$
\left(n_{2} / n_{1}\right) \bar{k}_{1} d=N \pi \text {. }
$$

For the destructive interference and a transmission minimum, it must be equal to a half-integer number of cycles

$$
\left(n_{2} / n_{1}\right) \bar{k}_{1} d=\left(N+\frac{1}{2}\right) \pi \text {. }
$$

These results agree with the positions of the maxima and minima of the transmitted fraction of the incident light intensity $\left|t\left(k_{1}\right)\right|^{2}$ in Eq. (1.2).

However, in addition to this, if we assume that the amplitude modulations of the various partial transmissions overlap spatially, there is also an interference between these amplitude modulations. This second interference modifies the effective velocity of the wave packet through the thin film and is the subject of this current study. When $\bar{k}_{1}$ is at the condition for a transmission maximum and each partial transmission occurs with the same phase, the overlapping amplitude modulations add constructively and give a greater weight to the region behind the relative maximum of the first partial transmission. This pushes the average position of the entire wave packet back and produces a slower effective velocity of the wave packet through the film. Likewise, when $\bar{k}_{1}$ is at the condition for a transmission minimum and each partial transmission occurs with alternating phase, the overlapping amplitude modulations add destructively and give a smaller weight to the region behind the relative maximum of the first partial transmission. This pulls the average position of the entire wave packet forward and produces a faster effective velocity of the wave packet through the film.

To be more quantitative, we consider the case where $A_{i}\left(k_{1}\right)$ is sufficiently sharply peaked about $\bar{k}_{1}$ so that

$$
\Delta k_{1}<\bar{k}_{1} \text {. }
$$

Experimentally this condition is easy to satisfy for visible light since it requires that the atomic transitions which produce the light are long compared to $10^{-16} \mathrm{~s}$. For such a case, the average position of the transmitted wave packet of
Eq. $(2.5)$ is $^{5.6}$

$$
x=\left(c / n_{1}\right) t-\delta^{\prime}\left(\bar{k}_{1}\right) \text {, }
$$

where from Eq. (1.3) the delay is given by

$$
\delta^{\prime}\left(\bar{k}_{1}\right)=\left\{\left[\left(n_{1}^{2}+n_{2}^{2}\right) / 2 n_{1}\right]\left|t\left(\bar{k}_{1}\right)\right|^{2}-1\right\} d .
$$

To obtain the effective velocity of the wave packet through the film, we note that the transmitted wave packet is peaked about the point $x=d$ at the time $t=(d / 2 c)\left(n_{1}^{2}+n_{2}^{2}\right)\left|t\left(\bar{k}_{1}\right)\right|^{2}$. The effective group velocity of the wave packet passing through the film region is then

$$
v_{\text {eff }}=\frac{2 c}{n_{1}^{2}+n_{2}^{2}}\left|t\left(\bar{k}_{1}\right)\right|^{-2},
$$

which takes on its maximum value

$$
\left(v_{\text {eff }}\right)_{\max }=c\left[\left(n_{1}^{2}+n_{2}^{2}\right) /\left(2 n_{1}^{2} n_{2}^{2}\right)\right]
$$

at transmission minima and which takes on its minimum value

$$
\left(v_{\text {eft }}\right)_{\min }=c\left[2 /\left(n_{1}^{2}+n_{2}^{2}\right)\right]
$$

at transmission maxima in analogy to the case of a scattering resonance in quantum mechanics.

At the maximum and minimum transmission conditions, the effective velocity of the light wave packet is symmetric under the interchange of $n_{1}$ and $n_{2}$. Whether the optically denser medium is the exterior region or the film, the effective velocity of the light wave packet will be the same. For example, for a thin film whose index of refraction is 1.5 and is surrounded by air, the wave speeds of a single plane wave in the air and in the film are $c$ and $c / 1.5$, respectively. However, for a wave packet of light, the effective speed within the optically dense film ranges between $c /$ 1.625 and $c / 1.385$ depending on whether there is maximum or minimum transmission, respectively. Conversely, if one had an air wedge between two pieces of the denser medium again the effective speed of a wave packet of light within the air wedge varies between $c / 1.625$ and $c / 1.385$ and would not be $c$ as expected for a monochromatic plane wave.

This same type of effective velocity variation occurs for the reflected portion of the incident wave packet as well. The reflected light consists of two components, the light reflected from the near surface of the film, i.e., the prompt reflection ( $p$ ); and the light which enters the film, makes an infinite series of partial reflections within it, and is transmitted through it in the backward direction, i.e., the delayed reflection (d). Again we expect that the lag of the delayed component behind the prompt component will depend on the interference character of the light-film system. Specifically, the prompt reflected amplitude is

$$
A_{r}^{\left(P^{\prime}\right.}\left(k_{1}\right)=A_{1}\left(k_{1}\right)\left[\left(n_{1}-n_{2}\right) /\left(n_{1}+n_{2}\right)\right] \text {, }
$$

the $j$ th delayed reflected component is

$$
\begin{aligned}
A_{,}^{(j)}\left(k_{1}\right)= & A_{i}\left(k_{1}\right)\left[4 n_{1} n_{2} /\left(n_{1}+n_{2}\right)^{2}\right] \\
& \times\left[\left(n_{2}-n_{1}\right) /\left(n_{2}+n_{1}\right)\right]^{2 j-1} e^{2 i k_{2} d j},
\end{aligned}
$$

and the total delayed component is

$$
\begin{aligned}
A_{r}^{(d)}\left(k_{1}\right)= & \sum_{j=1}^{\infty} A_{r}^{(j)}\left(k_{1}\right)=-A_{i}\left(k_{1}\right)\left(\frac{n_{1}-n_{2}}{n_{1}+n_{2}}\right) \\
& \times\left|t\left(k_{1}\right)\right| e^{2 i n_{2} k_{1} d / n_{1}} e^{i \epsilon\left(k_{1}\right)},
\end{aligned}
$$

where $\epsilon\left(k_{1}\right)$ is the delayed reflection phase shift above and 
beyond that due to the optical path length of the film,

$$
\epsilon\left(k_{1}\right)=\arctan \left(\frac{n_{1}^{2}+n_{2}^{2}}{2 n_{1} n_{2}} \tan \frac{n_{2} k_{1} d}{n_{1}}\right)-\frac{n_{2} k_{1} d}{n_{1}} .
$$

For the light produced by an atomic transition of a single atom, if the prompt reflection amplitude peaks at the position

$$
x_{p}=-c t \text {, }
$$

then, as in the treatment of the transmitted wave packet, the delayed reflection amplitude peaks at

$$
x_{d}=-c t+\left(2 n_{2} d / n_{1}\right)+\epsilon^{\prime}\left(\bar{k}_{1}\right) \text {, }
$$

where from Eq. (2.17), the extra delay is

$$
\epsilon^{\prime}\left(\bar{k}_{1}\right)=\left\{\left[\left(n_{1}^{2}+n_{2}^{2}\right) / 2 n_{1}^{2}\right]\left|t\left(\bar{k}_{1}\right)\right|^{2}-\left(n_{2} / n_{1}\right)\right\} d \text {. }
$$

This extra amount of the delay maximizes at

$$
\epsilon_{\max }^{\prime}=\left\{\left[\left(n_{1}^{2}+n_{2}^{2}\right) / 2 n_{1}^{2}\right]-\left(n_{2} / n_{1}\right)\right\} d
$$

for the transmission maxima and minimizes at

$$
\epsilon_{\min }^{\prime}=\left\{\left[2 n_{2}^{2} /\left(n_{1}^{2}+n_{2}^{2}\right)\right]-\left(n_{2} / n_{1}\right)\right\} d
$$

at transmission minima.

\section{EXPERIMENTAL CONSIDERATIONS}

Although the advance or delay of the transmitted wave packet might be able to be measured interferometrically by a beam division and recombination experiment with the thin film placed in one of the paths, a possibly simpler arrangement which achieves the same result is to observe the light reflected from a thin film. As the thickness of the film is increased, the coherence between the prompt and delayed reflections is gradually lost and the observed light intensity no longer exhibits bright and dark interference fringes. The usual explanation of this is that the beam which is delayed as it passes through the film lags further and further behind the prompt beam until their respective wave packets no longer overlap. ${ }^{7,8}$ The fact that the delay oscillates due to the changing interference character of the light-film system should be reflected as some sort of structure to the decrease in the reflected beam coherence. At transmission maxima, where the light is traveling slower than normal and the delay is longer than normal, coherence is lost at a shorter $d$ and the fringe visibility should be less than normal. At transmission minima, where the light is traveling faster than normal and the delay is shorter than normal, coherence is lost at longer $\boldsymbol{d}$ and the fringe visibility should be greater than normal

There are two statistical complications which tend to obscure the visibility of this variable lag of the delayed reflection. For any realistic light source, one is confronted with many atoms incoherently de-exciting at different times. Also, due to the slowness of our visual system compared to the period of oscillation of visible light, we observe the time-averaged intensity. For the reflected beam situation, the time-averaged intensity is

$$
\begin{aligned}
\langle I\rangle= & \int_{0}^{\infty} d k_{1} \mid A_{i}\left(k_{1}\right)\left(\frac{n_{1}-n_{2}}{n_{1}+n_{2}}\right)\left\{1-\left|t\left(k_{1}\right)\right|\right. \\
& \left.\times \exp \left(2 i n_{2} k_{1} d / n_{1}\right) \exp \left[i \epsilon\left(k_{1}\right)\right]\right\}\left.\right|^{2} .
\end{aligned}
$$

Making the substitution

$$
z \equiv k_{1}-\bar{k}_{1} \text {, }
$$

and by considering a strongly peaked frequency spectrum $A_{i}\left(k_{1}\right)$, Eq. (3.1) may be rewritten in a form analogous to the treatment of the time-averaged intensity and the coherence length given in Ref. 7:

$$
\begin{aligned}
\langle I\rangle \equiv & P-C(d) \cos \left(2 n_{2} \bar{k}_{1} d / n_{1}\right) \\
& +S(d) \sin \left(2 n_{2} \bar{k}_{1} d / n_{1}\right),
\end{aligned}
$$

where

$$
\begin{gathered}
P \equiv \int_{-\infty}^{\infty} d z\left(\frac{n_{1}-n_{2}}{n_{1}+n_{2}}\right)^{2}\left|A_{i}(z)\right|^{2}\left(1+|t(z)|^{2}\right), \\
C(d) \equiv 2 \int_{-\infty}^{\infty} d z\left(\frac{n_{1}-n_{2}}{n_{1}+n_{2}}\right)\left|A_{i}(z)\right|^{2}|t(z)| \\
\quad \times \cos \left[\left(2 n_{2} z d / n_{1}\right)+\epsilon(z)\right]
\end{gathered}
$$

and

$$
\begin{aligned}
S(d) \equiv & 2 \int_{-\infty}^{\infty} d z\left(\frac{n_{1}-n_{2}}{n_{1}+n_{2}}\right)\left|A_{i}(z)\right|^{2}|t(z)| \\
& \times \sin \left[\left(2 n_{2} z d / n_{1}\right)+\epsilon(z)\right] .
\end{aligned}
$$

The functions $C(d)$ and $S(d)$ are the real and imaginary parts of what is often-called the mutual coherence function. ${ }^{9}$

The major difference between these results and the treatment by Born and Wolf in Ref. 7 is the absence of the $\epsilon(z)$ term in their Eq. $(114)$ for $C(d)$ and $S(d)$ :

$$
\begin{aligned}
C_{B W}(d)= & 2 \int_{-\infty}^{\infty} d z\left(\frac{n_{1}-n_{2}}{n_{1}+n_{2}}\right)\left|A_{i}(z)\right|^{2} \\
& \times|t(z)| \cos \left(2 n_{2} z d / n_{1}\right)
\end{aligned}
$$

and

$$
\begin{aligned}
S_{B W^{\prime}}(d)= & 2 \int_{-\infty}^{\infty} d z\left(\frac{n_{1}-n_{2}}{n_{1}+n_{2}}\right)\left|A_{i}(z)\right|^{2} \\
& \times|t(z)| \sin \left(2 n_{2} z d / n_{1}\right) .
\end{aligned}
$$

In Eqs. (3.7) and (3.8), $C_{B W^{\prime}}(d)$ and $S_{B W^{\prime}}(d)$ are the Fourier cosine and sine transforms of the intensity spectrum of the light source and as such are reasonably smoothly varying functions of $d$. However, in Eqs. (3.5) and (3.6) the $C(d)$ and $S(d)$ functions have small oscillations superimposed on the smooth behavior. At a transmission maximum or minimum, a Taylor series expansion of $\epsilon(z)$ gives

$$
\begin{aligned}
C(d) \approx & 2 \int_{-\infty}^{\infty} d z\left(\frac{n_{1}-n_{2}}{n_{1}+n_{2}}\right)\left|A_{i}(z)\right|^{2} \\
& \times|t(z)| \cos \left\{\left[\left(2 n_{2} d / n_{1}\right)+\epsilon_{0}^{\prime}\right] z\right\} .
\end{aligned}
$$

For the case of a transmission maximum, the argument of the sine and cosine functions rises to a factor of $\left(n_{1}+n_{2}\right)^{2} /$ $4 n_{1} n_{2}$ above that of Eqs. (3.7) and (3.8), so that

$$
C(d)=C_{B W}\left\{\left[\left(n_{1}+n_{2}\right)^{2} / 4 n_{1} n_{2}\right] d\right\} .
$$

Similarly, at a transmission minimum, the argument of the sine and cosine functions in Eqs. (3.5) and (3.6) falls to a factor of $\left(n_{1}+n_{2}\right)^{2} / 2\left(n_{1}^{2}+n_{2}^{2}\right)$ below that of Eqs. (3.7) and (3.8), giving

$$
C(d)=C_{B W}\left(\left\{\left(n_{1}+n_{2}\right)^{2} /\left[2\left(n_{1}^{2}+n_{2}^{2}\right)\right]\right\} d\right) .
$$

The variability of the speed of the light wave packet within the film and the resulting lag of the delayed reflection caused by the interference character of the light-film system makes its presence known as this small oscillatory structure in the $C(d)$ and $S(d)$ functions. The spatial period 
of these small oscillations is of the order of $10^{-4} \mathrm{~cm}$, while the falloff lengths of $C(d)$ and $S(d)$ themselvesare typically a few centimeters or more. For the case of an uncomplicated atomic transition which produces a Gaussian intensity spectrum,

$$
\left[C(d)-C_{B W}(d)\right] / C_{B W}(d)=f_{\max } \approx O(-0.03)
$$

at a transmission maximum and

$$
f_{\min } \approx O(+0.9)\left|f_{\max }\right|
$$

at a transmission minimum. The usual measured quantity for determining the coherence length of a light beam is Michelson's fringe visibility function

$$
V(\bar{d})=\left(I_{\max }-I_{\min }\right) /\left(I_{\max }+I_{\min }\right) \text {, }
$$

where $\bar{d}$ is the average of the $d$ values which correspond to $I_{\max }$ and $I_{\min }$. For a symmetric frequency spectrum $A_{i}(z)$, $V(\bar{d})$ is proportional to the absolute value of $C(d)$ averaged between adjacent maximum and minimum intensity positions. The results of Eqs. (3.10)-(3.13) show that for a Gaussian intensity spectrum, the average of $C(d)$ at maximum and minimum intensity is a few tenths of a percent less than the average of $C_{B W}(d)$ at maximum and minimum intensity. This would make the fringe visibility function fall slightly faster than in the treatment given by Born and Wolf ${ }^{7}$ and should make the measured coherence length be slightly shorter than that obtained by independent measurements of $A_{i}(z)$. Expressed in an equivalent way, the slower velocity of the delayed wave packet and the resulting longer lag at transmission maxima cause the reflected minima to be less coherent and thus not as dark as expected. Similarly, the faster velocity of the delayed wave packet and the resulting shorter lag at transmission minima cause the reflected maxima to be more coherent and thus brighter than expected. Since these two perturbations do not average to zero, $V(\bar{d})$ is slightly smaller than expected.

\section{SUMMARY}

As mentioned in Sec. I, the familiarity that we have with the free-propagation of monochromatic light may serve as poor intuition when the propagation is not free or the light is not monochromatic. In this situation both limitations conspire to alter the group velocity of a wave packet of light from the expected value of $c / n$. This has nothing to do with the possible dispersion of the medium in which the light is propagating. It is solely a boundary condition plus nonmonochromaticity result. It dictates that the effective velocity of the wave packet of light through the film depends on the thickness of the film and the resulting interference character of the light.

'J. R. Reitz, F. J. Milford, and R. W. Christy, Foundations of Electromagnetic Theory (Addison-Wesley, Reading, MA, 1979), 3rd ed., Sec. 18-5. 'M. Born and E. Wolf, Principles of Optics (Pergamon, Oxford, 1970), 4th ed., Sec. 7.6.1

${ }^{3}$ Instead of relating the wave vectors in regions one and two to the wave vector in a vacuum, as is usually done, we relate the wave vector in region two to the wave vector in region one.

${ }^{4}$ A. Goldberg, H. M. Schey, and J. L. Schwartz, Am. J. Phys. 35, 177 (1967).

'E. P. Wigner, Phys. Rev. 98, 145 (1955).

${ }^{6}$ This result may also be obtained simply for the special case of a Gaussian frequency spectrum if (i) one assumes that $\bar{k}_{1}$ is such as to produce a transmission maximum or minimum, (ii) one approximates $t$ by an upward or inverted Gaussian centered on this value of $\bar{k}_{1}$, (iii) one Taylor series expands $\delta\left(k_{1}\right)$ about $\bar{k}_{1}$, and (iv) one performs the resulting Gaussian integrals analytically.

M. Born and E. Wolf, Ref. 2, Sec. 75.8.

${ }^{\mathrm{N}} \mathrm{F}$. A. Jenkins and H. E. White, Fundamentals of Optics (McGraw-Hill, New York, 1957), 3rd. ed., Sec. 13.12.

${ }^{9}$ L. Mandel and E. Wolf, Rev. Mod. Phys. 37, 231 (1965). 